\title{
Entanglement and the Nonlinear Elastic Behavior of Forests of Coiled Carbon Nanotubes
}

\author{
V. R. Coluci, ${ }^{1, *}$ A. F. Fonseca, ${ }^{2}$ D. S. Galvão, ${ }^{1}$ and C. Daraio ${ }^{3}$ \\ ${ }^{1}$ Instituto de Física "Gleb Wataghin,” Universidade Estadual de Campinas, C.P. 6165, 13083-970 Campinas SP, Brazil \\ ${ }^{2}$ Alan G. MacDiarmid NanoTech Institute, University of Texas, Richardson, Texas 75083-0688, USA \\ ${ }^{3}$ Aeronautics and Applied Physics, California Institute of Technology, Pasadena, California 91125, USA
}

(Received 28 August 2007; published 29 February 2008)

\begin{abstract}
Helical or coiled nanostructures have been objects of intense experimental and theoretical studies due to their special electronic and mechanical properties. Recently, it was experimentally reported that the dynamical response of a foamlike forest of coiled carbon nanotubes under mechanical impact exhibits a nonlinear, non-Hertzian behavior, with no trace of plastic deformation. The physical origin of this unusual behavior is not yet fully understood. In this Letter, based on analytical models, we show that the entanglement among neighboring coils in the superior part of the forest surface must be taken into account for a full description of the strongly nonlinear behavior of the impact response of a drop ball onto a forest of coiled carbon nanotubes.
\end{abstract}

DOI: 10.1103/PhysRevLett.100.086807

PACS numbers: 81.05.Uw, 81.05.Zx, 81.07.De

The study of nanostructures in special carbon nanotubes (CNTs) and nanowires has been the object of intense experimental and theoretical investigations due to their large range of possible applications and new physical phenomena [1,2]. Among these nanostructures, helical and coiled formations have a special place due to their differentiated mechanical behavior [3]. Coiled carbon nanotubes (CCNTs) were first predicted to exist in the early 1990s by Dunlap [4] and Ihara et al. [5-7] and experimentally observed in 1994 by Zhang et al. [8]. CCNTs have been receiving increasing interest because of their additional capability to serve as nanoscale mechanical springs [9] and electrical inductors [10], and for their potential applications in composites [11].

Recently, the dynamical response of a foamlike forest of CCNTs [Fig. 1(a)] under impact of a drop ball has been reported [12]. The experiment consisted of producing arrays of bundles of CCNTs [13,14], letting a stainless steel bead fall down on the forest of CCNTs, and measuring the dynamic force at the wall below the forest during the stages of penetration and restitution. The analysis of the forest's morphology after impact has shown no trace of plastic deformation and a full recovery of the foamlike layer of CCNTs under various impact velocities.

The contact force exhibits a strongly nonlinear dependence on displacement and appears fundamentally different from the response of a forest of CNTs [15-18]. The obtained results in [12] have been compared to the Hertz elastic model [19] of a solid sphere and a planar surface in contact where, if $F$ is the force of contact and $\delta$ is the displacement, $F \sim \delta^{1.5}[19]$. The results reported in Ref. [12] showed a nonlinear response of the CCNT forests having a force-displacement relation of $F \sim \delta^{2.2}$, different from the Hertzian case. It has been hypothesized that sideways interactions of the compressed CCNTs can be associated with the strong nonlinear behavior in CCNT forests, but the physical mechanisms behind it are still unclear.

In this Letter, we propose a model for the impact response of a forest of CCNTs. The model takes into ac-

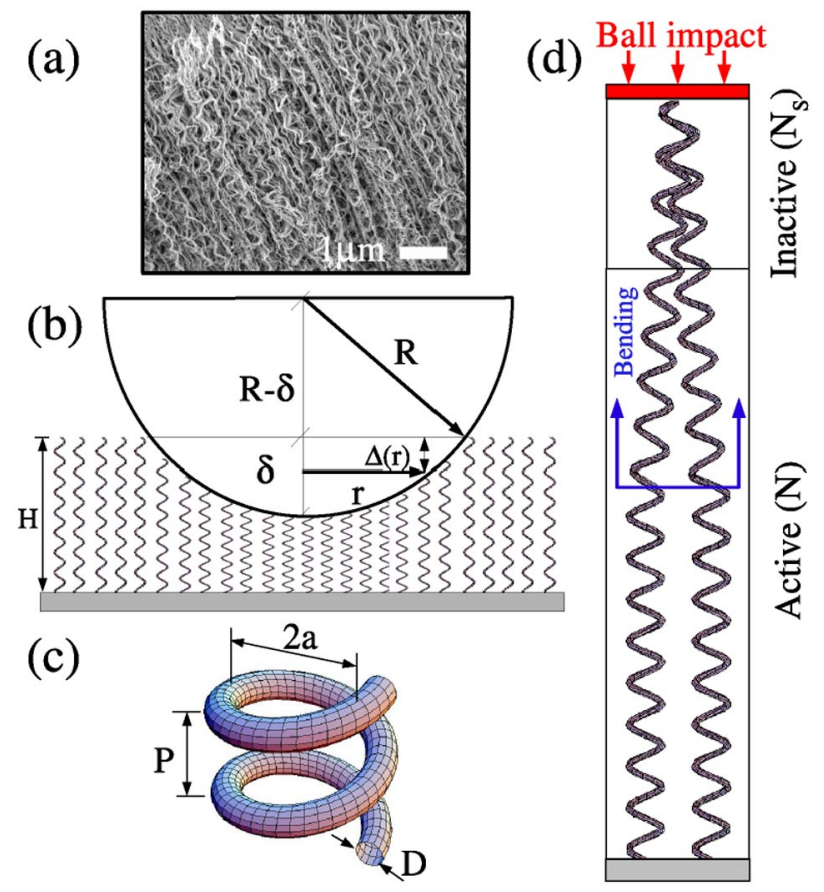

FIG. 1 (color online). (a) Scanning electronic microscopy picture of a CCNT forest [12]. (b) Schematic diagram of a CCNT forest deformed by a drop ball. $H$ is the forest thickness, $R$ is the ball radius, and $\delta$ is the total depth displacement of the ball into the forest. $\Delta$ is the axial deformation of the spring at a distance $r$ from the center of the contact between the ball and the forest. (c) Parameters of a helical spring: $a$ is the coil radius, $P$ is the spring pitch, and $D$ is the spring diameter. (d) Schematic representation of the entanglement between two adjacent coil bundles forming inactive, or "solid," and active turns. 
count: (i) the individual elastic contribution from each CCNT in contact with the drop ball; (ii) the geometry of the surface of contact between the forest and the drop ball; and (iii) sideways interactions of the compressed CCNTs through an entanglement process. As discussed below, we show that this model can describe the strongly nonlinear behavior observed in the recent experiments with CCNT forests [12].

In the model, the CCNT forest of thickness $H$ is considered as a set of identical springs that individually interact with the drop ball and that may laterally interact with other springs. The drop ball is considered to have a rigid spherical surface of radius $R$. Figure 1(b) illustrates the penetration of the ball into the CCNT forest and the geometric features of the model. The total depth displacement of the ball into the CCNTs forest is $\delta$. Each bundle of CCNTs is modeled as a helical spring of pitch $P$ with a Hooke's constant $k=\left(G D^{4}\right) /\left(64 a^{3} N\right)$ [20], where $G$ is the shear modulus, $N$ is the number of active turns, $a$ is the coil radius, and $D$ is the bundle diameter [Fig. 1(c)]. Typical values from experiment are $H=100 \mu \mathrm{m}, R=1000 \mu \mathrm{m}$, $2 a=0.45 \mu \mathrm{m}, \quad D=0.1 \mu \mathrm{m}, \quad \delta=3 \mu \mathrm{m}, \quad$ and $P=$ $0.9 \mu \mathrm{m}[12,14]$. We also considered the situation where the bundles of CCNTs are very close to each other. From Ref. [12], the total density of CCNTs in the forest is $100 / \mu \mathrm{m}^{2}$. Considering an ordered square grid and accounting for each bundle to be composed by $\sim 25$ tubes [12], this roughly gives an estimative of $0.56 \mu \mathrm{m}$ as the separation between two coiled bundles axis (and a $0.1 \mu \mathrm{m}$ outer distance between them). Therefore, it is reasonable to assume the existence of a certain degree of entanglement between some parts of the CCNTs throughout the forest which would contribute to sideway interactions. Furthermore, it is probable that changes in the entanglement occur during the dynamic contact between the ball and the forest surface. This mechanism is illustrated in Fig. 1(d), where it is shown that the impact of the ball on the forest top surface would cause a bending of the tips of each CCNT, leading to contacts among the superior turns of the adjacent coils. The entanglement mechanism can be very complicated and a long-range process. In the present model, the effect of this mechanism in the forest response is translated into the reduction of the number of active turns of each CCNT, i.e.,

$$
N=N_{T}-N_{S},
$$

where $N_{T}$ is the total number of turns of each CCNT $\left(N_{T}=\right.$ $H / P \cong 110$ ), and $N_{S}$ is the number of turns that become inactive due to the entanglement process [Fig. 1(d)]. The inactive turns form a "solid" phase, deriving from a complex entangled network of tubes, which is assumed not to contribute to the elastic response of the CCNT. This approach has been used by Rodrigues et al. [21] to obtain a nonlinear relation between the force and displacement of a conic spring. Based on the small displacement $(\sim 3 \mu \mathrm{m})$ of the top forest surface compared to the forest thickness $(\sim 100 \mu \mathrm{m})$, we also assume a short-range effect of the entanglement, i.e., only nearest neighboring coils interact. Initial entanglement prior to the impact can be incorporated in the initial number of active turns. The short-range interaction is included into the model by making the rate of increase of the number of inactive turns during the contact between the drop ball and the forest surface proportional to the ball velocity:

$$
\frac{d N_{S}}{d t}=\eta v,
$$

where $\eta$ is a measure of how many turns become inactive per unit length of the displacement. Despite the complexity of the interaction that forms the entanglement, $\eta$ is considered here constant and determined from experimental data a posteriori.

Since $v d t=d z$, where $z$ is the direction perpendicular to the forest surface (that is the direction of the movement of the ball), Eq. (2) can be easily integrated to give the following expression for the number of active turns:

$$
N(\Delta)=N_{T}-\eta \Delta,
$$

where $\Delta$ is the axial deformation of each CCNT. Figure 1(b) shows how to determine the value of $\Delta$ as a function of $\delta$ and the distance $r$ from the center of the contact between the ball and the CCNTs forest (hereafter called "center of contact" for short). From geometrical considerations, we have

$$
\Delta(r)=\sqrt{R^{2}-r^{2}}-(R-\delta) .
$$

The total force $F$ consists of a summation of the forces of each spring that interacts with the drop ball:

$$
F=k \sum_{i=0}^{M} n_{i} \frac{\Delta\left(r_{i}\right)}{1-\left(\eta / N_{T}\right) \Delta\left(r_{i}\right)},
$$

where $i$ represents the set of $n_{i}$ springs that are at the same distance $r_{i}$ from the center of contact, and $k$ is the effective spring constant of a single bundle of CCNTs with all $N_{T}$ turns active. At the edge of the contact area, $M$ is such that $\Delta\left(r_{M}\right)=0$, i.e.,

$$
M=\frac{\sqrt{2 R \delta-\delta^{2}}}{2 a} .
$$

We assume a circular symmetry of the projection of the surface of contact between the ball and the CCNTs forest, on the plane of the forest. Therefore, $n_{i}$ is given by

$$
n_{0}=1 \quad \text { and } \quad n_{i}=\frac{2 \pi r_{i}}{2 a}=2 \pi i, \quad i=1,2,3, \ldots, M,
$$

where we used that $r_{i}=2 a i$. The index $i=0$ is for the first coil hit by the ball. Equation (7) is, of course, an approximation for the situation where the concentration of the CCNTs is such that they are beside each other. Equa- 
tion (7) will be a good estimative of the number of coils at distance $r_{i}$ of the center of contact if the penetration of the ball is enough to ensure that many coils are hit by the drop ball. That is the case considered in the experiment, where a total circular contact area has a radius of $\sim 77 \mu \mathrm{m}$ and the CCNT bundle radius is $\sim 0.2 \mu \mathrm{m}$ [12]. For smaller depths, Eq. (7) should be corrected for a better result.

Since $\delta / R \ll 1$, it is reasonable to use the following approximations: $\Delta(r) \cong \delta-r^{2} /(2 R)$ and $M \cong \sqrt{2 R \delta} /(2 a)$. Thus, Eq. (5) can be rewritten as

$$
F \cong \frac{k \delta}{1-\left(\eta / N_{T}\right) \delta}+2 \pi k \sum_{i=1}^{M} \frac{i\left(\delta R-2 i^{2} a^{2}\right)}{\left[1-\left(\eta / N_{T}\right) \delta\right] R+2 \eta i^{2} a^{2}} .
$$

A simpler expression for $F$ cannot be derived from Eq. (8). However, by neglecting the term $2 \eta i^{2} a^{2}$ from the denominator, the following expression can be obtained,

$$
F=k \frac{\pi R \delta^{2}}{4 a^{2}\left[1-\left(\eta / N_{T}\right) \delta\right]},
$$

where terms proportional to $\delta / R$ were also neglected.

The result of these approximations to the final behavior of $F$ is shown in Fig. 2 using typical experimental parameters. We can see that this approximation is physically sound for $\eta \lesssim 10 / \mu \mathrm{m}$. We will see that this condition is satisfied in the experiments [12].

Using Eq. (9) to fit the experimental data [12], we obtain $k=1.988 \mathrm{~N} / \mathrm{m}$ and $\eta=7.066 / \mu \mathrm{m}$. The comparison with experiment is presented in Fig. 3.

The importance of the entanglement for the nonlinear behavior of the forest of CCNTs can be tested by recalculating the total force with $\eta=0$ in Eq. (3), yielding to
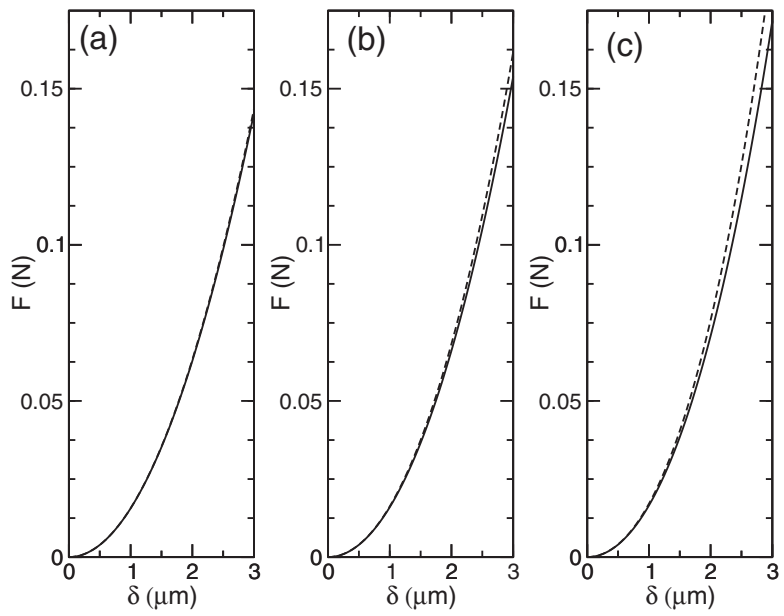

FIG. 2. Force-displacement curves obtained from Eq. (8) when the term $2 \eta i^{2} a^{2}$ is present (solid lines, no approximation) or not (dashed lines) for (a) $\eta=1 / \mu \mathrm{m}$, (b) $\eta=5 / \mu \mathrm{m}$, and (c) $\eta=10 / \mu \mathrm{m}$. The following parameters were used: $a=$ $0.225 \mu \mathrm{m}, R=1000 \mu \mathrm{m}, N_{T}=110$, and $k=1 \mathrm{~N} / \mathrm{m}$.

$$
F=k \frac{\pi R \delta^{2}}{4 a^{2}}
$$

Equation (10) shows that the force $F$ is proportional to $\delta^{2}$. Even being close to the relation found in [12], this result still does not capture the full nonlinear behavior of the impact response of a forest of CCNTs. However, when the entanglement is turned on again, our model, with the fitted parameters $k$ and $\eta$, recovers the previous experimental fitting, $F=A \delta^{m}, A=0.031$, and $m=2.2$ [12], which shows that the entanglement formed by lateral deformations of the CCNTs is necessary to explain the full strongly nonlinear behavior of the impact response of a drop ball onto a forest of CCNTs.

In a loading experiment, Cheng et al. have measured the spring constant value of a single amorphous carbon nanocoil in a low-strain regime (nanocoil elongation $\lesssim 3 \mu \mathrm{m}$ ) as being $0.12 \mathrm{~N} / \mathrm{m}$ [9]. The number of turns of that nanocoil is about 10 which leads to $\sim 1.2 \mathrm{~N} / \mathrm{m}$ for the value of the spring constant of a single turn. Using the derived $k$ value of the present model from experimental data [12], we can estimate the spring constant of a single turn of an individual CCNT. According to Ref. [12], the bundle is formed by $\sim 25$ nanocoils. Assuming that the bundle is formed by a parallel association of CCNTs, our estimative for the spring constant of a single CCNT is $k_{s}=k / 25 \cong$ $0.08 \mathrm{~N} / \mathrm{m}$. The spring constant of a single turn of the CCNT in a compression experiment is, then, $k_{s} N_{T}=$ $8.7 \mathrm{~N} / \mathrm{m}$, which is the same order of magnitude of the value for a single amorphous carbon nanocoil measured in a tension experiment.

As previously mentioned, $\eta$ is the number of turns that become inactive per unit length of displacement. Supposing that the entanglement is formed by inactive turns that

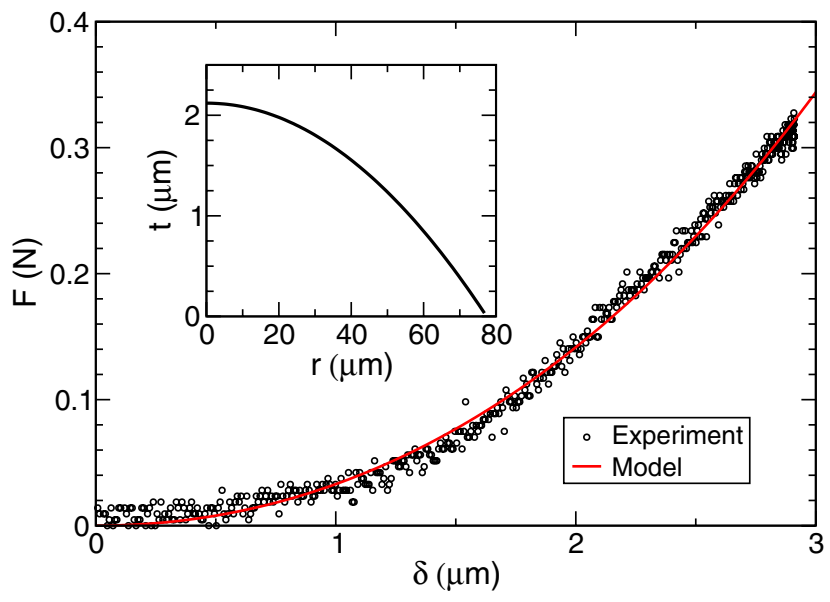

FIG. 3 (color online). Behavior of the force as function of the displacement of the drop ball during contact with the CCNT forest. The model curve was obtained using Eq. (9) with $k=$ $1.988 \mathrm{~N} / \mathrm{m}$ and $\eta=7.066 / \mu \mathrm{m}$. The inset graph shows the behavior of the thickness of the entanglement as a function of distance from the center of impact $(r=0)$. 
form a "solid" phase in the top of the CCNT forest, we can estimate the thickness, $t$, of the entanglement at a given distance $r$ from the center of impact as

$$
t(r)=\eta D \Delta(r),
$$

where $\Delta(r)$ is given by Eq. (4). The inset graph of Fig. 3 displays $t$ as a function of $r$. For the center of impact, the thickness is $2.1 \mu \mathrm{m}$, and the number of inactive turns of the coil at the center of impact is $\sim 21$, about $20 \%$ of the total number of turns of the total forest thickness. These results are compatible with what we expect for an entanglement formed by the superior parts of the CCNTs due to the impact of a drop ball on the forest.

If instead of a ball we have an approximated perfect cube or parallelepiped with a finite contact area $A$ with the forest, falling down on the forest of CCNTs, its impact response can be estimated using Eq. (5). In this case, all nanocoils feel the same axial deformation $\delta$, and the sum in Eq. (5) can easily be performed to give $F \sim n_{c} \delta /[1-$ $\left.\left(\eta / N_{T}\right) \delta\right]$, where $n_{c}$ is the number of nanocoils in contact with the face of the cube or parallelepiped, and is simply given by $n_{c} \sim A /\left(\pi a^{2}\right)$. The response force of the forest, then, will be approximately linear with $\delta$, for small $\delta$ values, and start growing nonlinearly for larger $\delta$ ones, as a consequence of the entanglement.

It should be stressed the limit of validity of our model [represented by Eq. (2)] takes into account the experimental condition [12] of small forest deformations. The contribution of the entangled part of the forest to the elastic response is neglected because any deformation of the entanglement is expected to be significantly smaller than the forest deformations. One of the predictions of the model that can be experimentally tested is the thickness value of the entanglement of the top forest surface. We hope the present Letter will stimulate further experiments to test the validity of the present model.

In conclusion, we have derived an analytical model for the nonlinear behavior of the impact response of a forest of CCNTs including geometrical and physical aspects during the forest compression. We showed that the nonlinear behavior is fully described when the entanglement of the coiled carbon nanotubes in the superior part of the forest surface is incorporated into the model. This entanglement among neighbors is due to the bending of the coil tips produced by the ball impact. Under the experimental conditions of small deformations [12], the model predicts an entanglement thickness of $\sim 2 \mu \mathrm{m}$ at the maximum forest compression. The model results point out to the importance of the coil entanglements for the elastic behavior of such systems. The present model is able to provide, by matching experimental values, estimates of the spring constant of a single CCNT and the level of entanglement between CCNTs. These aspects can play an essential role in the future design of micro-electro-mechanical systems devices, new shock protecting layers, and composites for microelectronic packaging and vibration mitigating materials where CCNT structural entanglement could be present.

We acknowledge the financial support from IMMP/ MCT, IN/MCT, BNN/CNPq, THEO-NANO, and the Brazilian agencies FAPESP, CAPES, and CNPq. A. F. F. acknowledges additional support from the CNPq.

*Author to whom correspondence should be addressed.

FAX: +55-19-35215376.

coluci@ifi.unicamp.br

[1] S. Iijima, Nature (London) 354, 56 (1991).

[2] R.H. Baughman, A.A. Zakhidov, and W.A. de Heer, Science 297, 787 (2002).

[3] A. F. da Fonseca and D. S. Galvão, Phys. Rev. Lett. 92, 175502 (2004) and references cited therein.

[4] B. I. Dunlap, Phys. Rev. B 46, 1933 (1992).

[5] S. Itoh, S. Ihara, and J. Kitakami, Phys. Rev. B 47, 1703 (1993).

[6] S. Itoh, S. Ihara, and J. Kitakami, Phys. Rev. B 48, 5643 (1993).

[7] S. Itoh and S. Ihara, Phys. Rev. B 48, 8323 (1993).

[8] X. B. Zhang, X. F. Zhang, D. Bernaerts, G. T. Vantendeloo, S. Amelinckx, J. Vanlanduyt, V. Ivanov, J. B. Nagy, P. Lambin, and A. A. Lucas, Europhys. Lett. 27, 141 (1994).

[9] X. Chen, S. Zhang, D. A. Dikin, W. Ding, R.S. Ruoff, L. Pan, and Y. Nakayama, Nano Lett. 3, 1299 (2003).

[10] S. Motojima, X. Chen, S. Yang, and M. Hasegawa, Diam. Relat. Mater. 13, 1989 (2004).

[11] K. T. Lau, M. Lu, and D. Hui, Composites. Part B, Engineering 37, 437 (2006).

[12] C. Daraio, V. F. Nesterenko, and S. Jin, J. Appl. Phys. 100, 064309 (2006).

[13] W. Wang and A. M. Rao, Bulk Synthesis of Helical Coiled Carbon Nanostructures (Materials Research Society, Boston, MA, 2005), p. HH.2.8.1.

[14] P. R. Bandaru, C. Daraio, K. Yang, and A. M. Rao, J. Appl. Phys. 101, 094307 (2007).

[15] H. J. Qi, K. B. K. Teo, K. K.S. Lau, M. C. Boyce, W. I. Milne, J. Robertson, and K. K. Gleason, J. Mech. Phys. Solids 51, 2213 (2003).

[16] S.Dj. Mesarovic, C.M. McCarter, D.F. Bahr, H. Radhakrishnan, R.F. Richards, C.D. Richards, D. McClain, and J. Jiao, Scr. Mater. 56, 157 (2007).

[17] C. Daraio, V.F. Nesterenko, J. AuBuchon, and S. Jin, Nano Lett. 4, 1915 (2004).

[18] C. Daraio, V.F. Nesterenko, and S. Jin, Appl. Phys. Lett. 85, 5724 (2004).

[19] L.D. Landau and E. M. Lifshitz, Theory of Elasticity (Pergamon, Oxford, 1986).

[20] K. Gjerde, J. Kjelstrup-Hansen, C. H. Clausen, K. B. K. Teo, W. I. Milne, H.G. Rubahn, and P. Boggild, Nanotechnology 17, 4917 (2006).

[21] E. Rodrigues, M. Paredes, and M. Sartor, J. Mech. Des. 128, 1352 (2006). 